\title{
Avaliação do perfil de consumo, armazenamento e descarte de medicamentos por indivíduos que frequentam farmácias privadas em Jaraguá do Sul - SC
}

\author{
Evaluation of the consumption profile, storage and disposal of medicines by individuals who attend \\ private pharmacies in Jaraguá do Sul - SC \\ Evaluación del perfil de consumo, almacenamiento y eliminación de medicamentos por parte de \\ personas que ateden farmácias privadas em Jaraguá do Sul - SC
}

\section{Resumo}

O avanço da tecnologia farmacêutica proporcionou um aumento na fabricação de medicamentos, o que tem contribuído para o aumento da automedicação e descarte inadequado. Este estudo teve como objetivo avaliar a automedicação, armazenamento e descarte de medicamentos em pacientes de farmácias privadas de Jaraguá do Sul, SC. Para isto, aplicou-se um questionário com clientes que frequentam farmácias privadas do município. O questionário possuía 32 perguntas, divididas em 4 etapas: dados sociodemográficos, prevalência de automedicação e o perfil de consumo de medicamentos, conhecimento sobre armazenamento, validade e integridade dos medicamentos e o conhecimento sobre o descarte correto e o impacto causado ao meio ambiente. Com os resultados obtidos de 120 pacientes, foi possível observar que $91,7 \%$ deles se automedicam. Além disso, observou-se que $65 \%$ nunca receberam orientações sobre como descartar os medicamentos vencidos ou em desuso. Por isso, $58 \%$ dos entrevistados descartam de forma inadequada, e estudos evidenciam que quando em contato com o solo e a água, esses medicamentos podem causar contaminação, que mesmo em uma rede de tratamento de esgoto não é eliminada completamente. É um problema pouco divulgado, porém com consequências catastróficas. Com os resultados do presente estudo, conclui-se que a população tem sua parcela de responsabilidade também devendo estar esclarecida e envolvida no processo de conscientização do uso, armazenamento e da geração de resíduos derivados dos medicamentos, reforçando a necessidade de estratégias de educação sobre o tema.
\end{abstract}

Palavras-chave: Medicamentos; Automedicação; Armazenamento; Meio ambiente.

\begin{abstract}
The advancement of pharmaceutical technology demonstrated an increase in the manufacture of medicines, which has contributed to the increase in self-medication and inappropriate disposal. This study aimed to evaluate selfmedication, storage and disposal of medicines in patients from private pharmacies in Jaraguá do Sul, SC. Therefore, a questionnaire was applied to customers who attend private pharmacies in the municipality. The questionnaire had 32
\end{abstract}


questions, divided into 4 stages: sociodemographic data, prevalence of self-medication and the profile of medication consumption, knowledge about the storage, validity and integrity of the drugs and knowledge about the correct disposal and the impact caused to the environment. With the results obtained from 120 patients, it was possible to observe that $91.7 \%$ of them are self-medicating. In addition, it was observed that $65 \%$ never received guidance on how to dispose of expired or out-of-use medications. For this reason, 58\% of respondents discarded inappropriately, and studies show that when in contact with soil and water, these drugs can cause contamination, which even in a sewage treatment network is not completely eliminated. With the results of the present study, we concluded that the population has its share of responsibility and must also be informed and involved in the process of raising awareness of the use, storage and generation of residues derived from medicines, reinforcing the need for education strategies on the subject.

Keywords: Medicines; Self-medication; Storage; Environment.

\section{Resumen}

El avance de la tecnología farmacéutica ha dado lugar a un aumento de la fabricación de medicamentos, lo que ha contribuido al aumento de la automedicación y la eliminación inadecuada. Este estudio tuvo como objetivo evaluar la automedicación, almacenamiento y eliminación de medicamentos en pacientes de farmacias privadas en Jaraguá do Sul, SC. Para ello, se aplicó un cuestionario a los clientes que acuden a las farmacias privadas del municipio. El cuestionario contó con 32 preguntas, divididas en 4 etapas: datos sociodemográficos, prevalencia de automedicación y perfil de consumo de medicación, conocimiento sobre el almacenamiento, validez e integridad de los medicamentos y conocimiento sobre la correcta disposición y el impacto causado al medio ambiente. Con los resultados obtenidos de 120 pacientes se pudo observar que el 91,7\% de ellos se automedican. Además, se observó que el 65\% nunca recibió orientación sobre cómo desechar los medicamentos vencidos o fuera de uso. Por este motivo, el 58\% de los encuestados los descartan de forma inapropiada, y los estudios muestran que al entrar en contacto con el suelo y el agua, estos medicamentos pueden provocar contaminación, que incluso en una red de tratamiento de aguas residuales no se elimina por completo. Es un problema poco publicitado, pero con consecuencias catastróficas. Con los resultados del presente estudio, se concluye que la población tiene su parte de responsabilidad y también debe estar informada e involucrada en el proceso de sensibilización sobre el uso, almacenamiento y generación de residuos derivados de medicamentos, reforzando la necesidad de educación. estrategias sobre el tema.

Palabras clave: Medicamentos; Automedicación; Almacenamiento; Medio ambiente.

\section{Introdução}

Estima-se que cerca de 30 mil toneladas de medicamentos são jogadas fora no lixo comum ou vasos sanitários pelos consumidores a cada ano no Brasil (Vaz et al., 2011). Apesar de seu uso ser fundamental para a manutenção da saúde da população, a facilidade de aquisição e o incentivo da mídia geram um uso excessivo de medicamentos e com isso, o acúmulo nas residências (Ferreira et al., 2005). No Brasil pelo menos 35\% dos medicamentos adquiridos são feitos através de automedicação (Aquino, 2008).

O uso indiscriminado de medicamentos tornou-se um dos grandes problemas enfrentados pela saúde no âmbito mundial, uma vez que é apontado como uma das principais causas de intoxicações humanas registradas no país (Lessa et al., 2008). Vale ressaltar que, as condições inadequadas de uso (administração incorreta, dose inadequada ou não necessidade do medicamento) e armazenamento (locais inadequados, medicamentos vencidos) podem afetar sua efetividade e segurança, gerando problemas de saúde, como enfermidades iatrogênicas e mascaramento de doenças evolutivas (Sharif et al., 2010).

Em decorrência da sobra de medicamentos, os mesmos acabam sendo descartados de forma inadequada, fato esse preocupante, uma vez que podem causar contaminação ao meio ambiente e afetar a saúde da população (Vaz et al., 2011). No Brasil por exemplo, o sistema de esgoto não está apto para a realização de tratamentos adequados de resíduos tóxicos incluindo os medicamentos descartados de modo incorreto (Vaz et al., 2011; Santos et al., 2016). Esse é um grande problema, uma vez que diversas classes terapêuticas como os estrógenos, já foram detectadas nos rios, e mostraram potencial de afetar a reprodução dos organismos aquáticos, além de que fármacos ainda biologicamente ativos, podem estar presentes em peixes consumidos por seres humanos. Já foi observado também que a mistura de diferentes medicamentos no meio ambiente, pode causar uma toxicidade mais pronunciada, quando comparado ao fármaco administrado isoladamente podendo apresentar efeitos adversos e tóxicos desconhecidos (Ribeiro et al, 2019. Nesse sentido, os medicamentos não devem ter a mesma destinação final de resíduos comuns (Vaz, Freitas \& Cirqueira, 2011). Apesar de existirem normativas técnicas sobre o 
descarte de medicamentos (resolução 306/2004 e a 358/2005), elas dizem respeito aos estabelecimentos de saúde, não incluindo a população, o que dificulta a compreensão dos efeitos prejudiciais do descarte inadequado (Brasil, 2004; 2005).

Considerando que existe uma parcela considerável da população que realiza automedicação e mantém as farmácias caseiras bem como a falta de conhecimento sobre a forma adequada de armazenamento e descarte de medicamentos, torna-se relevante a realização de estudos que identifiquem o conhecimento da população sobre a automedicação, armazenamento e descarte correto de medicamentos. Dessa forma, o objetivo do presente trabalho foi avaliar o conhecimento de pacientes atendidos em farmácias privadas de Jaraguá do Sul, SC sobre automedicação, armazenamento e descarte consciente de medicamentos.

\section{Metodologia}

Foi realizado um estudo exploratório, transversal, com uma abordagem quantitativa (Hair et al., 2005), baseado em entrevistas, realizado no período de 28 de junho a 03 de julho de 2021, na cidade de Jaraguá do Sul, Santa Catarina. Os participantes foram abordados em farmácias e drogarias privadas do município.

A coleta de dados foi realizada por meio de entrevistas individuais e orientada por um questionário estruturado contendo questões objetivas, conforme evidenciado na Tabela 1. Antes de iniciar o questionário, os participantes foram orientados sobre os objetivos da pesquisa, e após os esclarecimentos, houve a leitura e assinatura do termo de consentimento livre e esclarecido.

$\mathrm{O}$ questionário foi dividido em quatro etapas. Na primeira etapa da entrevista foram investigados os dados sociodemográficos (gênero, idade, estado civil, nível de escolaridade, área de trabalho, estrutura familiar, entre outros). Na segunda etapa, foi avaliada a prevalência de automedicação e o perfil de consumo de medicamentos. Além disso, foi avaliada a utilização de medicamentos recomendados por terceiros, o uso de medicamentos sem prescrição e o uso correto, interação medicamentosa e efeitos adversos. Na terceira etapa, os indivíduos foram interrogados sobre o armazenamento, validade e integridade dos medicamentos. Por último, foi avaliado o conhecimento sobre o descarte correto e o impacto causado ao meio ambiente pelo descarte incorreto de medicamentos.

Os dados obtidos pelas entrevistas foram tabulados e analisados utilizando o programa Excel®. Os resultados foram expressos em frequência relativa e absoluta. O projeto de pesquisa foi aprovado pelo Comitê de Ética de Pesquisa com seres humanos (Parecer 4.803.655). A presente pesquisa seguiu os preceitos do Código de Ética de Pesquisa com seres humanos, conforme a resolução 466/2012, garantindo o respeito e autonomia dos participantes, a justiça e equidade (Brasil, 2012).

\section{Resultados e Discussão}

Foram entrevistados 120 indivíduos, atendidos em farmácias e drogarias privadas do município de Jaraguá do Sul, SC. A Figura 1 mostra os dados sociodemográficos da população entrevistada (gênero, idade, estado civil, nível de escolaridade, área de trabalho, estrutura familiar). 
Figura 1. Dados sociodemográficos da população entrevistada.

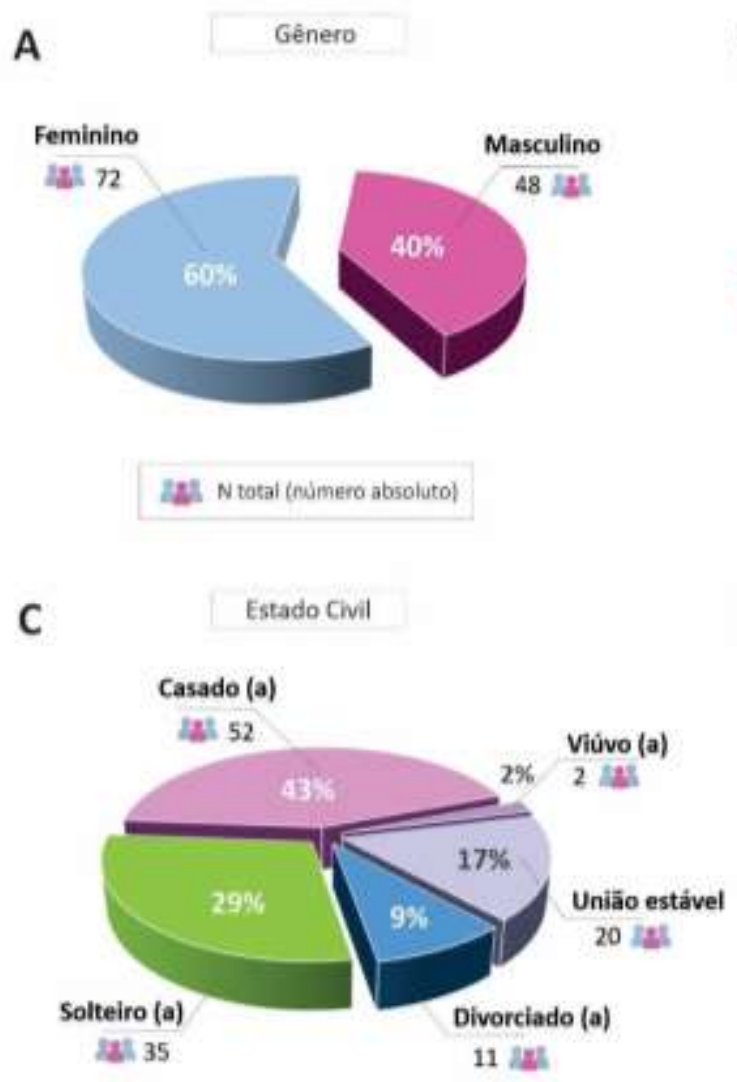

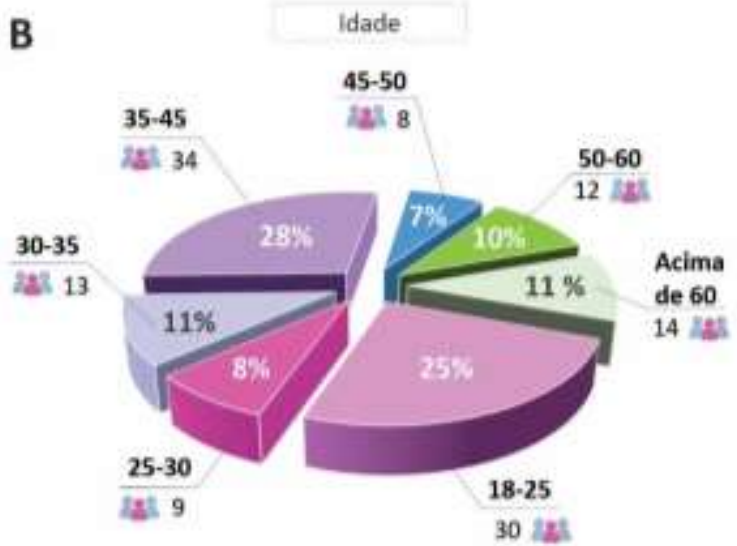

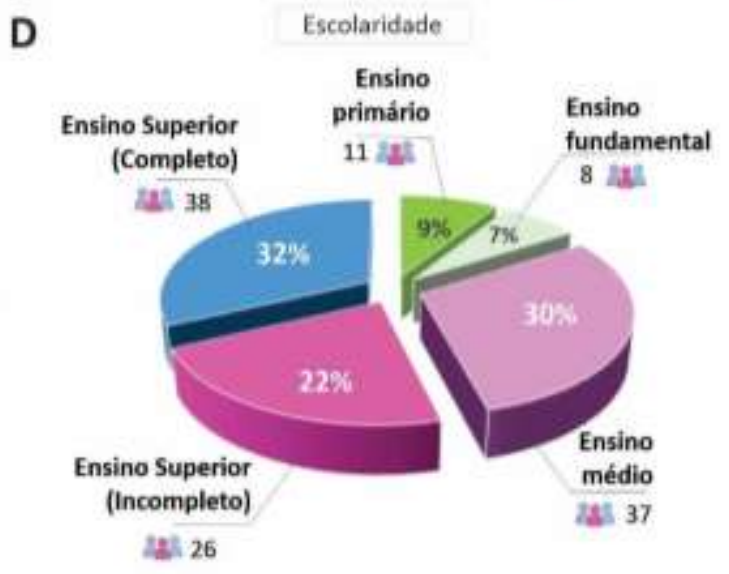

$\mathbf{E}$

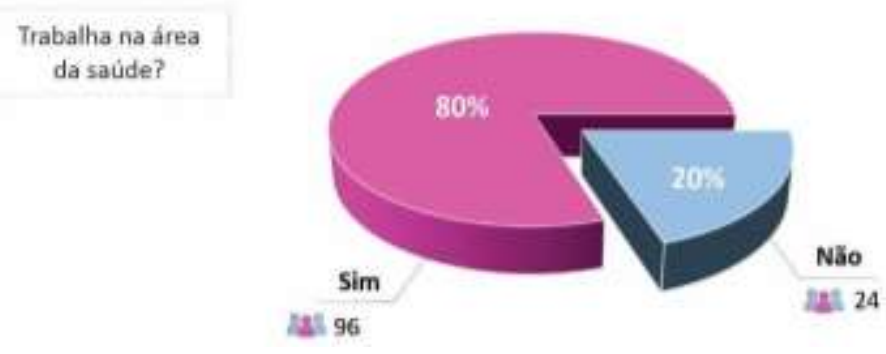

Fonte: Autoria própria com os dados coletados nas entrevistas.

De uma forma geral, observa-se que a maioria dos entrevistados é do sexo feminino, em uma faixa etária de 35 a 45 anos. A predominância do sexo feminino (60\%) provavelmente se deve ao fato de que as mulheres utilizam mais os serviços de saúde do município quando comparadas aos homens, e com isso acabam comprando mais medicamentos. Além disso, 20\% deles eram da área da saúde.

Quanto aos dados de automedicação, 95,8\% dos entrevistados responderam saber o significado desse termo e 91,7\% dos entrevistados relataram se automedicar. Desses, $16,7 \%$ se automedicam com frequência, 50,8\% às vezes e $24,2 \%$ raramente. Esses resultados estão de acordo com estudos que evidenciam que o Brasil é um dos principais consumidores mundiais de medicamentos (Dos Santos \& Ferreira, 2012). De fato, aspectos como o não-cumprimento da apresentação da receita médica, excesso de propagandas sobre medicamentos juntamente com a falta de informação e orientação na população em geral justificam a preocupação com a qualidade da automedicação praticada no país (Arrais et al., 1997). Na Tabela 1 podese observar que houve uma maior prevalência da automedicação em mulheres (58\%) quando comparado aos homens (38\%). O 
predomínio da automedicação em mulheres está de acordo com um estudo conduzido por Malta et al. (2017), na qual pacientes do sexo feminino foram as que mais relataram automedicação, e também com maior frequência, demonstraram preocupação com a saúde em relação aos homens. De fato, o consumo de medicamentos por automedicação é uma prática predominante entre as mulheres e menos frequente entre os homens. Segundo Arrais et al. (1997), a predominância do uso de medicamentos entre as mulheres ocorre parcialmente devido a propaganda de medicamentos, que relacionam os papéis sociais tradicionalmente atribuídos às mulheres, entre eles o de prover a saúde da família.

Tabela 1. Frequência relativa e absoluta de pacientes que se automedicam por sexo.

\begin{tabular}{|c|c|c|c|c|c|}
\hline & \multicolumn{2}{|c|}{ Sim } & \multicolumn{2}{|c|}{ Não } & \multirow[b]{2}{*}{ Total } \\
\hline & $\mathbf{N}^{\mathbf{o}}$ & $\%$ & $\mathbf{N}^{\mathbf{o}}$ & $\%$ & \\
\hline Masculino & 45 & 38 & 3 & 2,6 & 48 \\
\hline Feminino & 70 & 58 & 2 & 1,66 & 72 \\
\hline
\end{tabular}

Fonte: Dados coletados nas entrevistas expressos em dados brutos e em porcentagem.

No que se refere a compra de medicamentos (Figura 2), 73\% dos entrevistados relataram que costumam comprar medicamentos isentos de prescrição. Desses indivíduos foi possível observar que 73\% eram do sexo feminino, o que demonstra uma maioria de mulheres que apresentam esse comportamento.

Figura 2. Frequência de indivíduos que compram medicamentos sem prescrição por sexo.

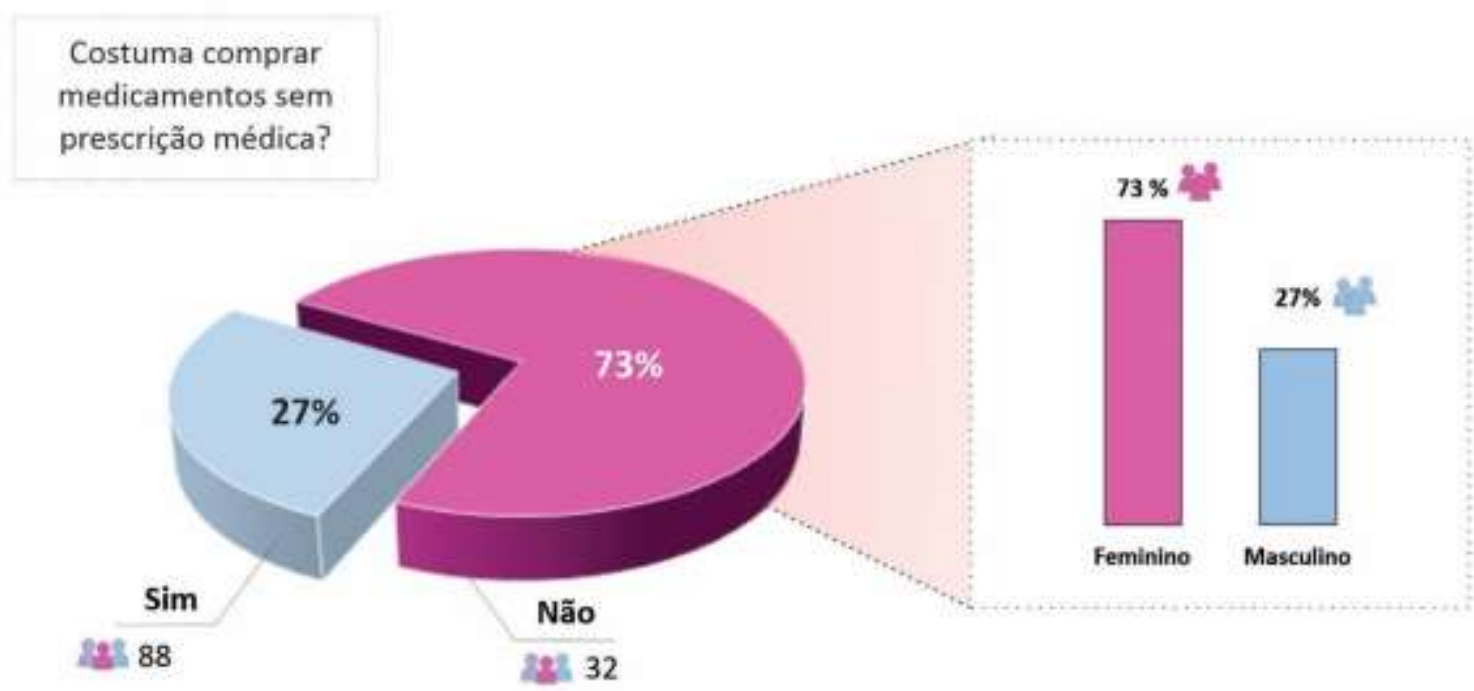

Fonte: Autoria própria com os dados coletados nas entrevistas.

De acordo com Girotto et al. (2010) a automedicação pode trazer benefícios como redução do tempo e custo, diminuição da busca pelos serviços públicos de saúde e maior comodidade e autonomia para o paciente no tratamento de transtornos menores. Porém, se administrados de forma inconsequente, a utilização de fármacos coloca em risco a saúde da população. Essa prática pode acentuar os riscos relacionados aos medicamentos prescritos, retardar o diagnóstico adequado e mascarar doenças (Santos et al., 2016). Nesse cenário, o papel do farmacêutico torna-se essencial, uma vez que é o último profissional da saúde a ter contato com o paciente, e pode orientá-lo sobre a real necessidade do uso de determinada medicação (Ely et al., 2015).

Ao questionar os entrevistados sobre consulta na bula a respeito de interações com outros medicamentos que o 
indivíduo já está utilizando, foi observado que $60 \%$ verificam as possíveis interações. No entanto, $40 \%$ dos entrevistados relataram não verificar as possíveis interações medicamentosas, um fato bastante preocupante uma vez que elas podem causar efeitos adversos e diminuir a eficácia dos medicamentos (Moura et al., 2009). Fonteles et al. (2009) mostraram que as mulheres apresentam maior percentual de efeitos adversos a medicamentos, provavelmente devido a fatores como, maior consumo de medicamentos, maior frequência às consultas médicas e maior adesão às prescrições médicas. Esse dado está de acordo com o presente estudo, uma vez que $17 \%$ dos entrevistados relataram ter apresentado algum efeito adverso à medicação, sendo que $80 \%(\mathrm{~N}=16)$ eram mulheres e $20 \%(\mathrm{~N}=4)$ eram homens.

A forma de armazenar medicamentos é sempre uma dúvida entre seus usuários, conforme representado na Tabela 2, é possível verificar locais onde são armazenados os medicamentos e como estes são preservados dos indivíduos entrevistados.

Tabela 2. Avaliação do armazenamento de medicamentos pelos entrevistados.

\begin{tabular}{lll} 
& $\mathbf{N}^{\mathbf{0}}$ & $\mathbf{\%}$ \\
Costuma armazenar medicamentos em casa? & 71 & 59 \\
Sim & 10 & 8 \\
Não & 16 & 13 \\
Apenas de uso contínuo & 23 & 19 \\
Apenas de uso frequente & & \\
Como você armazena os medicamentos? & 84 & 70 \\
Em caixas originais com bula & 2 & 2 \\
Em caixas originais sem bula & 24 & 20 \\
Todos na mesma caixa com identificação & 10 & 8 \\
Todos na mesma caixa sem identificação & & \\
Onde você costuma armazenar os medicamentos? & 55 & 46 \\
\hline Na cozinha & 25 & 21 \\
No quarto & 36 & 30 \\
Não tem lugar definido & 3 & 3 \\
\hline Sala & & \\
\hline
\end{tabular}

Fonte: Dados coletados nas entrevistas expressos em dados brutos e em porcentagem.

Estudos mostram que o local mais habitual de armazenar medicamentos é na cozinha, devido à acessibilidade do local, por geralmente estar perto de filtros de água e ou na presença de outros líquidos que podem ser ingeridos com o medicamento e de utensílios, como colheres para medida de soluções e suspensões (Santos \& Lopes, 2017). Os resultados observados neste trabalho corroboram com dados de uma pesquisa realizada por Schwingel et al. (2015), no qual 59\% dos participantes armazenavam os medicamentos na cozinha. É importante ressaltar que, o armazenamento de medicamentos em locais úmidos, com variações constantes de temperatura, como é o caso dos banheiros e da cozinha, pode interferir na eficácia dos medicamentos, em função de alterações em suas propriedades físico-químicas (Milanez et al., 2013).

Vale destacar que uma grande parcela dos entrevistados relatou verificar a data de validade (67\%) e o estado físico 
(75\%) dos medicamentos armazenados antes do consumo. Esses dados detalhados podem ser observados na Tabela 3 e estão de acordo com um do estudo de Piveta et al. (2015), que mostrou que $81,3 \%$ dos entrevistados armazenam os medicamentos em caixas originais com bula e mais da metade $(60,1 \%)$ verificar o prazo de validade dos medicamentos. No entanto, uma parcela considerável dos entrevistados (20\%) não verifica, ou fazem isso raramente em relação a data de validade dos medicamentos utilizados, e ainda, que cerca de $25 \%$ dos indivíduos responderam que não observam aspectos como cor, odor e integridade do medicamento antes de fazer o seu uso. Esses resultados são importantes, uma vez que o uso de medicamentos vencidos e/ou mal acondicionados podem ocasionar danos à saúde, como intoxicações e efeitos adversos mais acentuados (Marin et al., 2003). Além disso, podem ser um risco potencial ao meio ambiente, uma vez que seus resíduos quando expostos à temperatura e luz, podem transformar-se em substâncias tóxicas e afetar ciclos biogeoquímicos, afetando as cadeias alimentares (Eickhoff et al., 2009).

Tabela 3. Frequência e forma de avaliação das características de medicamentos.

\begin{tabular}{lll}
$\begin{array}{l}\text { Quando utiliza medicamento armazenado em casa, } \\
\text { costuma verificar a data de validade? }\end{array}$ & $\mathbf{N}^{\mathbf{o}}$ & $\mathbf{\%}$ \\
\hline Sim, sempre verifico & & 67 \\
Frequentemente verifico & 15 & 13 \\
Às vezes verifico & 17 & 14 \\
Quase nunca verifico & 8 & 6 \\
\hline $\begin{array}{l}\text { Você costuma observar o estado (cor, odor, integridade) } \\
\text { do medicamento armazenado antes de fazer uso? }\end{array}$ & & \\
\hline Sim & 90 & 75 \\
Não & 30 & 25 \\
\hline
\end{tabular}

Fonte: Dados coletados nas entrevistas expressos em dados brutos e em porcentagem.

Com relação ao descarte de medicamentos, na presente pesquisa, verificou-se que a grande maioria (65\%) não recebe informações sobre como descartar corretamente o medicamento vencido ou em desuso. O farmacêutico, como principal responsável envolvido em todas as ações associadas ao medicamento, deve incluir em suas responsabilidades a preocupação com o final do ciclo de vida dele, buscando tanto a segurança do paciente, como do meio ambiente (Medeiros \& Moreira, 2014). De acordo com os autores Boff e Rigon (2018), a população que descarta os medicamentos de forma incorreta, o fazem por não saber dos locais que recebem esses resíduos, falta de orientação dos órgãos responsáveis e devido a população considerar que apenas os órgãos públicos são responsáveis pelo descarte e esquecendo que os cidadãos também devem atuar em prol desta causa.

O presente estudo mostrou que $56 \%$ dos participantes afirmam saber como realizar o descarte correto de medicamentos. No entanto, foi observado um conflito quando foram questionados sobre os locais onde descartam os medicamentos (Figura 3), uma vez que 58\% dos entrevistados relataram descartar no lixo comum/pia/vaso sanitário, seguido de $41 \%$ que devolve/descarta onde adquiriu. Um estudo realizado por Fernandes et al. (2020) no município de DivinópolisMG, constatou que $74 \%$ das pessoas realizavam o descarte diretamente no meio ambiente, como lixo doméstico, vaso sanitário, pias da cozinha e/ou banheiro, rios e lagos. Uma pesquisa feita Santos \& Frizon (2019), realizada em Sananduva-RS, demonstrou que aproximadamente $76 \%$ dos entrevistados descartam os medicamentos de maneira inadequada, sendo que destes, $65 \%$ jogam no lixo comum. Os achados do presente estudo, em conjunto com outros mencionados, apenas reforçam a 
necessidade de orientação do paciente tanto pelo farmacêutico quanto por órgãos públicos.

Figura 3. Locais onde os indivíduos entrevistados descartam os medicamentos.

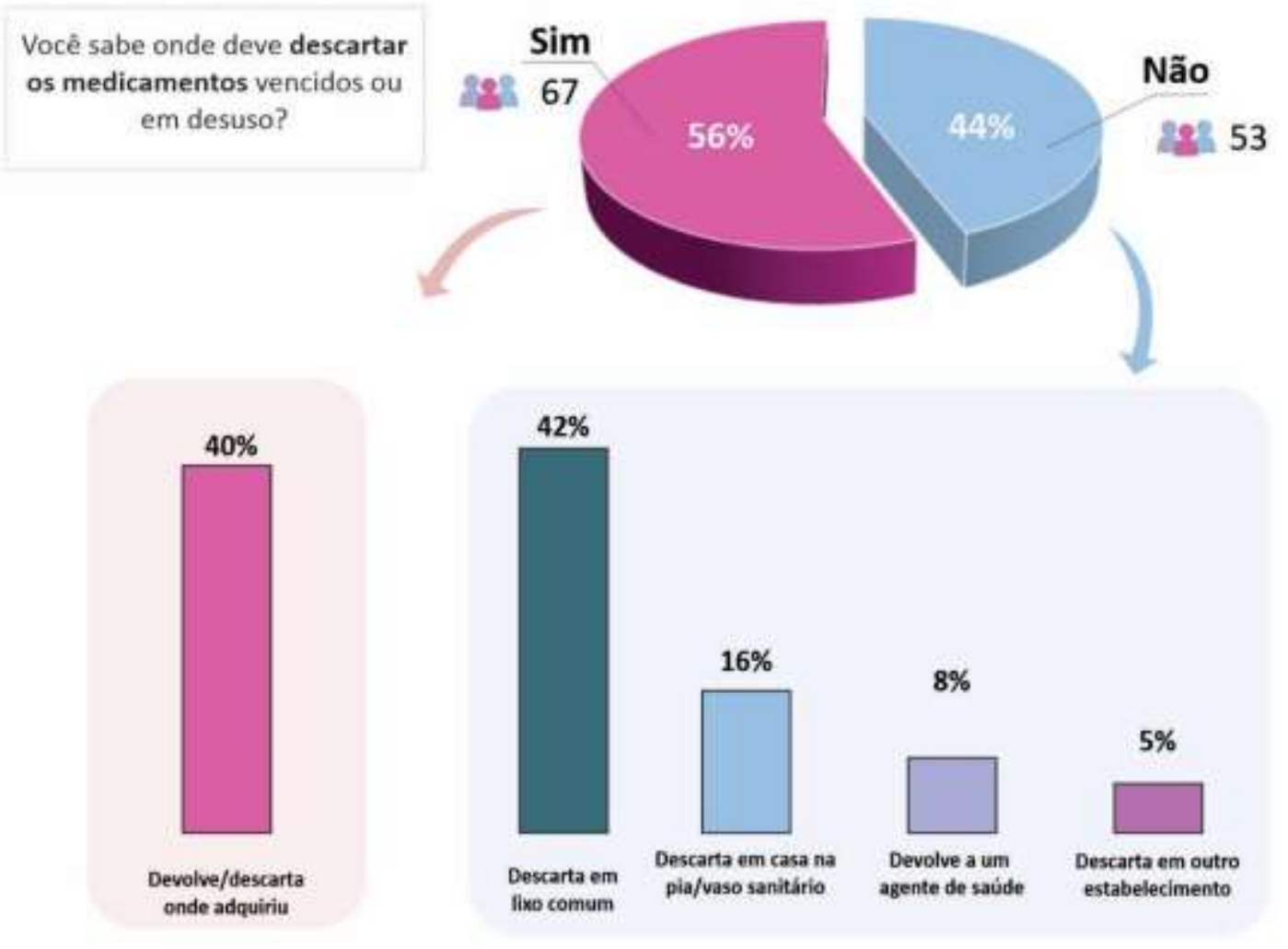

Fonte: Autoria própria com os dados coletados nas entrevistas.

Os usuários foram questionados sobre o que acontece com o medicamento após o descarte correto, a maioria (84\%) respondeu não saber. Além disso, 74\% dos entrevistados, desconhecem os impactos ambientais e na saúde de um descarte incorreto. Na Figura 4 pode se observar que dos entrevistados que afirmam conhecer o impacto do descarte incorreto (26\%), parte deles diz estar relacionado com danos ao meio ambiente, intoxicação de terceiros ou ainda resistência a medicamentos. Segundo Pinto et al. (2014) o descarte incorreto de medicamentos facilita que catadores consumam de forma inadequada os fármacos ou que descartem de maneira direta no solo, para reaproveitamento das embalagens. O contato destes trabalhadores com os resíduos farmacológicos caracteriza um risco à saúde, podendo causar intoxicações devido a possibilidade de reutilização dos medicamentos. 
Figura 4. Conhecimento dos entrevistados sobre os impactos do descarte incorreto.

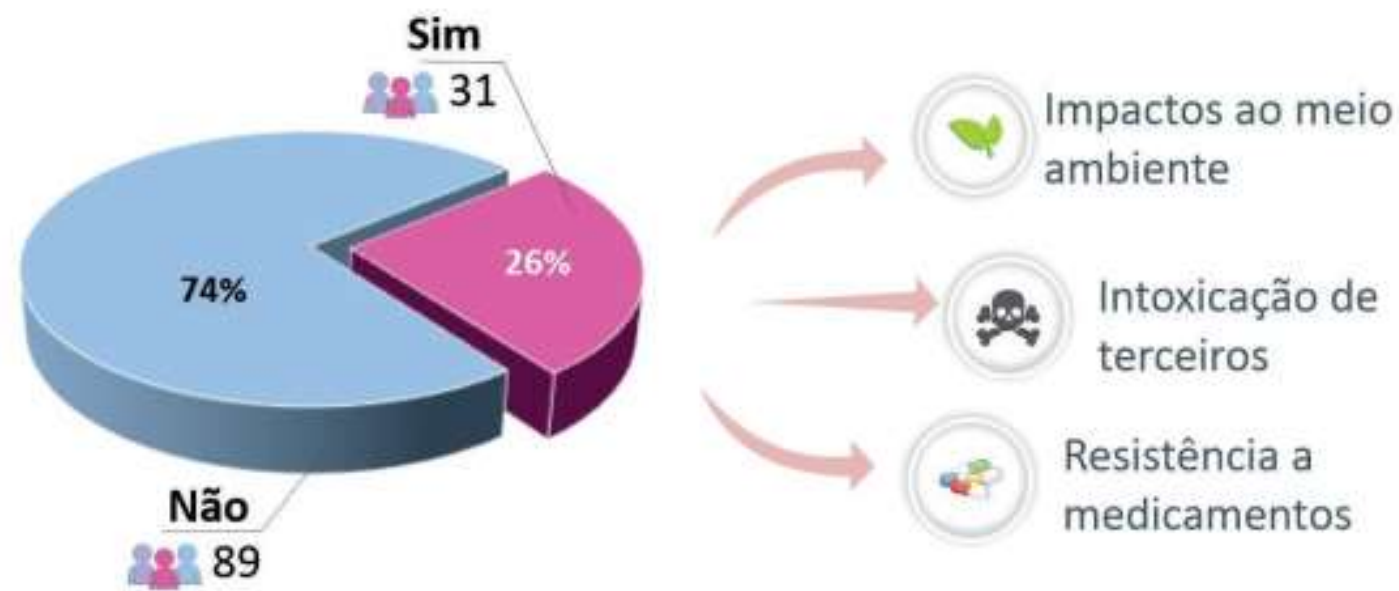

Fonte: Autoria própria com os dados coletados nas entrevistas.

Os medicamentos quando a céu aberto, são resíduos químicos que fazem parte do lixo e disseminam doenças através de vetores que utilizam esses resíduos como fonte de nutrientes para se desenvolver ou que se multiplicam nesses locais. Além disso, várias dessas substâncias não são completamente removidas nas Estações de Tratamentos de Esgoto, pois tem alto potencial para bioacumulação e baixa biodegradabilidade, de forma que muitos fármacos resistem aos vários processos de tratamento de água (Vaz et al., 2011; Pinto et al., 2014).

A preocupação com o ambiente vem crescendo e surgindo a preocupação de como se pode prevenir que dejetos prejudiciais sejam jogados no mesmo de maneira incorreta causando malefícios tanto ao solo quanto aos seres vivos que habitam esses ambientes como os animais e as pessoas (Oliveira et al., 2015).

\section{Conclusão}

Essa pesquisa revelou que ainda há muita falta de informação para a população sobre os perigos da automedicação e do armazenamento incorreto de medicamentos, que podem trazer danos diretos à saúde do indivíduo. Além disso, muitos não sabem a importância de descartar os medicamentos de forma adequada, o que pode levar ao aumento dos riscos de contaminação do meio ambiente e aumento da possibilidade de ingestão de resíduos farmacêuticos tóxicos por humanos e animais.

Portanto, considerando a necessidade de conscientização da população, cabe ao profissional farmacêutico, juntamente com as autoridades pertinentes sobre o assunto, tentarem suprir a falta de informação de grande parte da população quanto aos riscos da automedicação e uso desnecessário de medicamentos, importância do seu armazenamento correto a fim de garantir as propriedades físico-químicas, segurança e qualidade desses produtos bem como métodos e conduta adequada para seu descarte.

Vale ressaltar que mais estudos serão realizados a fim de avaliar esses dados em uma parcela maior da população do município de Jaraguá do Sul, além de outros municípios vizinhos, para que futuramente sejam realizadas ações com o objetivo de informar a população sobre os riscos associados aos medicamentos: desde seu consumo, armazenamento e descarte. 


\section{Referências}

Aquino, D. S. (2008). Por que o uso racional de medicamentos deve ser uma prioridade? Ciência e Saúde Coletiva. 13 , 733-6.

Arrais, P. S. D., Coelho, H. L. L., Batista, M. C. D. S., Carvalho, M. L., Righi R. E. \& Arnau, J. M. (1997). Perfil da automedicação no Brasil. Rev. Saúde Pública. 31(1), 71-7.

Berwanger, E. K. \& Júnior, C. D. A. (2018). Análise dos fatores que afetam a leitura é interpretação da bula em moradores do município de Cujubim-RO. Revista da Faculdade de Educação e Meio Ambiente - FAEMA, Ariquemes. 9(ed esp), 484-90

Bila, D. M. \& Dezotti, M. (2003). Fármacos no Meio Ambiente. Química Nova. 26 (4), 523-30

Boff, E. \& Rigon, D. (2018). Descarte de medicamentos realizados pela população de Santa Helena, SC. Unoesc \& Ciência. 9 (2), 139-46,

Dos Santos, E. C. \& Ferreira, M. A. (2012). A indústria farmacêutica e a introdução de medicamentos genéricos no mercado brasileiro. Nexos Econ. 6 (2). 95119.

Eickhoff, P. Heineck, I. \& Seixas, L.J. (2009). Gerenciamento e destinação final de medicamentos: uma discussão sobre o problema. Revista Brasileira de Farmácia. 90 (1), 64-8.

Ely, L.S. Engroff, P., Guiselli, S. R., Cardoso, G. C., Morrone, F. B. \& Carli G. A.(2015). Uso De Anti-inflamatórios e analgésicos por uma população de idosos atendida na Estratégia Saúde da Família. Revista Brasileira Geriatria e Gerontologia. 18 (3), 475 - 85.

Fernandes, M. R., Figueiredo, R. C. Silva, L. G. Rocha, R. S. \& Baldoni A. O. (2020). Armazenamento e descarte dos medicamentos vencidos em farmácias caseiras: problemas emergentes para a saúde pública. Einstein.18, 1-6

Ferreira, W. A. Silva, M. E. S. T. Paula, A. C. C. F. \& Resende, C. A. M. B. (2005). Avaliação da farmácia caseira no município de Divinópolis - MG por estudantes do curso de farmácia da UNIFENAS. Infarma. 17 (7/9), 84 -6.

Fonteles, M. M. F., Francelino, E. V., Santos, L. K. X., Silva, K. M., Siqueira, R., Viana, G. S. B., Vasconcelos, S. M. M., Sousa, F. C. F. \& Monteiro, M. P. (2009). Reações adversas causadas por fármacos que atuam no sistema nervoso: análise de registros de um centro de farmacovigilância do Brasil. Rev Psiquiat Clin. 36(4), 137-44.

Girotto, E. Matos, D. B. S. \& Oliveira, J. M. (2010). Perfil da automedicação em população residente de arapongas, Paraná. Revista espaço para a Saúde. Londrina. 11 (2), 29-38.

Hair, J.F. Babin, B. Money A.H. Samouel, P. (2005) Fundamentos De Metodos De Pesquisa Em Administracão. 1(ed).

Lessa M. A. \& Bochner, R. (2008). Análise das internações hospitalares de crianças menores de um ano relacionadas a intoxicações e efeitos adversos de medicamentos no Brasil. Revista Bras. Epidemiol. 11 (4), 660-74.

Malta, D. C., Bernal, R. T. I., Lima M. G., Araújo, S. S. C., Silva, M. M. A. D., Freitas, M. I. F. \& Barros, M. B. A. (2017). Noncommunicable diseases and the use of health services: analysis of the National Health Survey in Brazil. Rev Saúde Pública. 51, Supl 1:4s.

Marin, N. Castro, O. L. \& Machado, S. C. G. S. (2003). Assistência farmacêutica para gerentes municipais. Rio de Janeiro (RJ): OPAS/OMS.

Medeiros, M. S. Moreira, L. M. \& Lopes, C. C. (2014). Descarte de medicamentos: programas de recolhimento e novos desafios. Rev Cienc Farm Básica Apl. $35(4), 651-62$.

Milanez, M. C. Stutz, E. Rosales, T. O. Penteado, A. J. Perez, E. Cruciol, J. M., Pereira, E. V. \& Bovo F. (2013). Avaliação dos estoques domiciliares de medicamentos em uma cidade do Centro-Sul do Paraná. Rev Ciênc Méd Biol. 12(3), 283-9.

Moura, C. Acurcio, F. \& Belo N. (2009). Drug-drug interactions associated with length of stay and cost of hospitalization. J Pharm Pharmaceut Sci. 12(3), 266-72.

Oliveira, J. C. Lima, L. O. M. Zan, L. B. Marcondes, G. I. M. Ilha, M. Marques, L. A. M. (2015). Implantação de postos de coleta para o descarte adequado de medicamentos e subsequente destinação final. Rev Inter Face HS-Saúde, Meio Ambiente e Sustentabilidade. 10(1), 104-16.

Pinto, G. M. F. Silva, K. R. D. Pereira, R. D. F. A. B. \& Sampaio, S. I. (2014). Estudo do descarte residencial de medicamentos vencidos na região de Paulínia (SP), Brasil. Engenharia Sanitária e Ambiental. 19 (3), 219-24.

Piveta, L. N. Silva, L. B. Guidoni, C. M. \& Girotto E. (2015). Semina: Armazenamento e descarte de medicamentos por acadêmicos da área da saúde de uma universidade pública paranaense. Ciências Biológicas e da Saúde, Londrina. 36 (1), 55-66.

Resolução CONAMA n. 358, de 29 de abril de 2005. (2005). Dispõe sobre o tratamento e a disposição final dos resíduos dos serviços de saúde e dá outras providências. Publicação DOU, n. 084, de 04 de maio de 2005, p. 63-65.

Resolução n. 306, de 07 de dezembro de 2004. (2004). Dispõe sobre o regulamento técnico para o gerenciamento de resíduos de serviços de saúde. Ministério da Saúde.

Resolução n. 466, de 12 de dezembro de 2012. (2012). Conselho nacional de Saúde. 
Research, Society and Development, v. 10, n.9, e13210917917, 2021

(CC BY 4.0) | ISSN 2525-3409 | DOI: http://dx.doi.org/10.33448/rsd-v10i9.17917

Ribeiro, T. A. Silva, A. M. Morais, F. V. Gadretborio, V. Araujo, A. N. Ebram, P. \& Fernandes, W. S. (2019). Avaliação do descarte adequado de medicamentos vencidos e não utilizados no município de Jacareí-SP. Braz. J. Hea. Rev. 2 (5), 4876-82.

Santos, A. M., Carneiro L. S., Chaud, L. C. S., Urias, G. M. P. C. \& Brum H. C. C. (2016). Estudo do perfil farmacoterapêutico de pacientes idosos portadores de diabetes tipo II, Pindamonhangaba, SP. Revista de Ciência e Saúde. 1(3), 24-33.

Santos, R. C. \& Frizon, N. S. (2019). Descarte inadequado de medicamentos vencidos ou em desuso. R. gest. sust. ambient. 8(1), 290-300.

Santos, R. C. \& Lopes, M. L. (2017). A farmácia domiciliar e a utilização de medicamentos em residências da zona rural do município de Ubá (MG). Rev Cient Faminas. 12(2), 27-36.

Santos, S. L. F. Nogueira, T. B. K. B. Silva, P. R. M. \& Oliveira, F. R. A. M. (2016). Aspectos toxicológicos do descarte de Medicamentos: Uma questão de educação em saúde, 9(3), 7-20.

Schwingel, D. Souza, J. Simonetti, E. Rigo, M. P. Ely, L. S. Castro, L. C., Fernandes, C. \& Kauffmann, C. (2015). Farmácia caseira x Uso racional de medicamentos. Rev Cad Pedagógico. 12(3), 117-30.

Serafim, E. O. P. Del Vecchio, A. Gomes, J. Miranda, A. Moreno, A.H. Loffredo, L.M.C. Salgado, H.R.N. \& Chung M.C. (2007). Qualidade dos medicamentos contendo dipirona encontrados em residências de Araraquara e sua relação com a atenção farmacêutica. Revista Brasileira de Ciências Farmacêuticas. 43(1), 203-10.

Sharif, S. I., Abduelkarem, A. R., Bustami, H. A., Haddad, L. I. \& Khalil, D. S. (2010). Trends of Home Drug Storage and Use in Different Regions across the Northern United Arab Emirates. Rev. Med. Princ. 19, 355-8.

Vaz, K. V. Freitas, M. M. \& Cirqueira, J. Z. (2011). Investigação sobre a forma de descarte de medicamentos vencidos. Cenarium Pharmacêutico. 4 (4), 17. 20. 